\title{
Necker's Cube and the Universe
}

\author{
PAUL T E CUSACK*
}

Independent Researcher, BSc E, DULE, 1641 Sandy Point Rd, Saint John, NB, Canada E2K 5E8, Canada

*Corresponding Author: PAUL T E CUSACK, Independent Researcher, BSc E, DULE, 1641 Sandy

Point Rd, Saint John, NB, Canada E2K 5E8, Canada

Abstract: This paper deals with the Necker's cube Optical Illusion and how that associates with AT Math and the physical universe. The funnel and a Coriolis is considered in light of Necker's Cube. Knowledge of AT Math is presumed.

Keywords: Necker's Cube; Optical Illusion; AT Math; Astrotheology; Calirnaut; Poisson's ratio: Human $D N A$

\section{INTRODUCTION}

In the ordinary world, what we see is a $3 \mathrm{D}$ space converted to a $2 \mathrm{D}$ image on the retina. The brain interprets the 2D image on the retina as 3D space. In an optical illusion, such as the Necker's Cube, we go from a $2 \mathrm{D}$ image on paper to an imagined $3 \mathrm{D}$ space. However, the brain can and is tricked int the Necker Cube. We can see two varieties of an object in space from a 2D image.

When we apply simple geometry to this problem, we get that the universe is an illusion. The 2D image on the brain represents the physical universe. It can be seen in two ways. So which of the universe is correct? Does it matter?

\section{Necker's Cube}

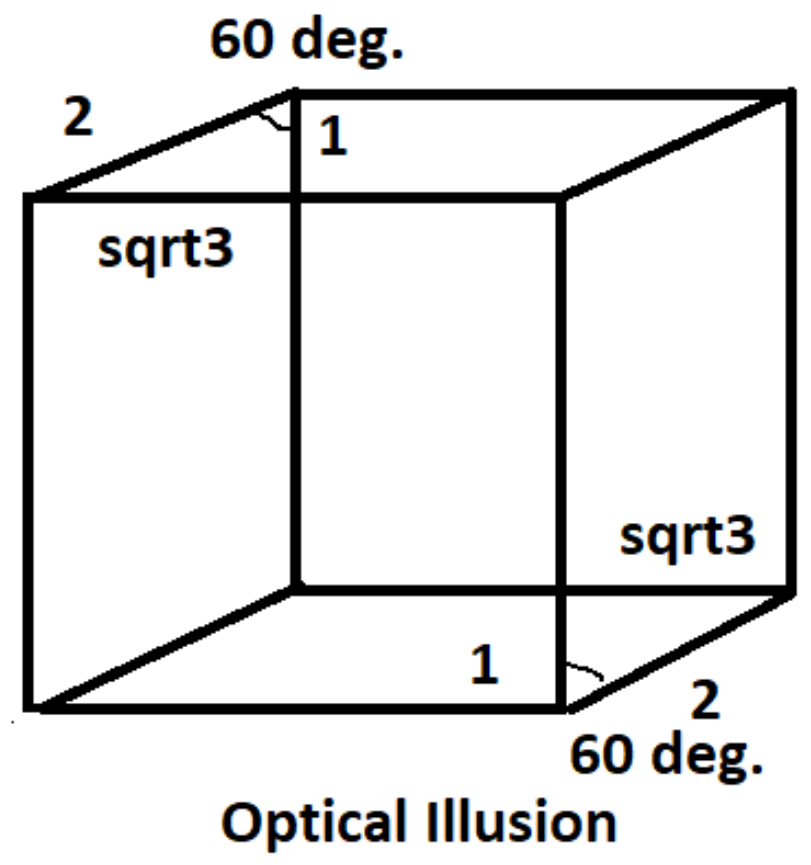

Figure1. 
Cube $_{1}$ R-L:

$\mathrm{s}=\mathrm{Et} \sin \theta$

$\mathrm{s} /(\mathrm{Et})=\sin 60^{\circ}$

$\mathrm{s} /(1)(1)=\sin 60^{\circ}$

$\mathrm{s}=\sin 60^{\circ}$

$\mathrm{s}=\mathrm{t}$

Cube $_{2}$ L-R

$\mathrm{s}=\mathrm{Et} \sin \theta$

$\mathrm{s}=\mathrm{Et} \sin 30^{\circ}=\cos 60^{\circ}$

$\mathrm{s}=1 / 2$

$\mathrm{s}_{2 / /} \mathrm{s}_{1}=(1 / 2) /(0.866)=\mathbf{1} / \sqrt{ } \mathbf{3}=\mathbf{E}$

$\mathrm{s}_{1} / \mathrm{s}_{2}=0.866 /(1 / 2)=\sqrt{3}=\mathbf{t}=$ Eigenvector

$\mathrm{E}=1 / \mathrm{t}$

$1 / \sqrt{ } 3=1 / \sqrt{ } 3$

But:

Cube $_{1}=$ Cube $_{2}$

$\mathrm{s}_{1}=\mathrm{s}_{2}$

$\sin 60^{\circ}=\sin 30^{\circ} \chi$

$0.866=1 / 2 \chi$

$\chi=\sqrt{ } 3$

Consider:

\section{Universal Parametric Equation:}

[csc $\left.60^{\circ} ; 3\right]$

$=(\mathrm{x}, \mathrm{y})=[\mathrm{E} ; \mathrm{t}]$

$\mathrm{E}=1 / \sin 60^{\circ} ; \mathrm{t}=3$

$\mathrm{s}=\mathrm{Etsin} \theta \times \sqrt{3}$

$=\left(1 / \sin 60^{\circ}\right)(3) \times \sqrt{ } 3$

$=6.00$

$\mathrm{S}=\mathrm{t}$

$1 / \mathrm{t}=1 / \mathrm{s}=\mathbf{1} / \mathbf{6}=\mathrm{E}=\mathrm{V}+$

$s=\left(1 / \sin 60^{\circ}\right)(\sqrt{ } 3) \times \sqrt{ } 3$

$=2598 \sim 0.26=$ Poisson's Ratio.

\section{Symmetrical Body:}

$200 \mathrm{lbs}=90.9 \mathrm{~kg} / 176 \mathrm{~cm}$ in height $=516$

$\mathrm{M}=\mathrm{Ln} \mathrm{t}=516=\mathrm{Ln} \mathrm{t}$

$\mathrm{t}=1676=1$ Dalton

x $2.15=360.38 \mathrm{gm}$

$360^{\circ} / 2=180^{\circ}$

$60^{\circ} \times 3=180^{\circ} \sin 6006=104.6 \sim 105$

International Journal of Scientific and Innovative Mathematical Research (IJSIMR) 
$\mathrm{t}=3: \mathrm{E}=5 \Rightarrow \mathrm{SE}=\mathrm{SE}$

The universe is chiral. It is both left and right-handed. Of course, your left hand is controlled by the right brain; and vice verse. So why is it left and right-handed? It is neither. It is both at once. The cube is

$2^{\wedge} 3=8$

$\mathrm{t}^{\wedge} 3=1 \mathrm{t}=+/-1$

They meet at $\mathrm{zt}=0$

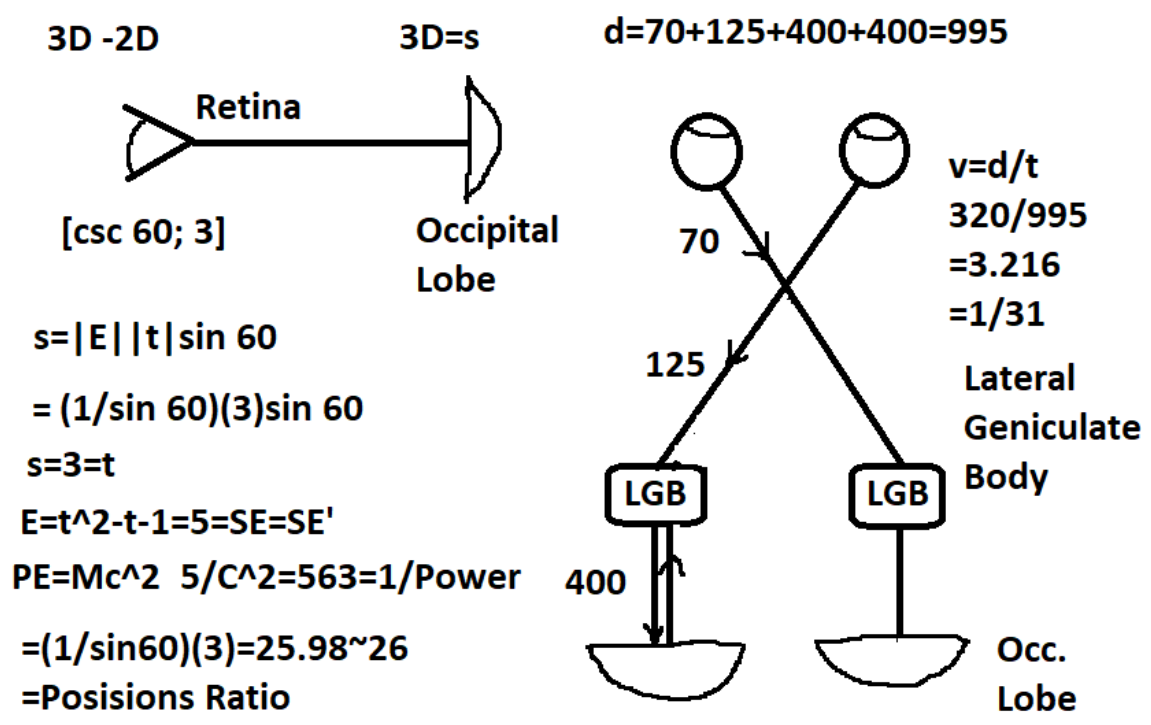

Figure2.

$$
\begin{aligned}
& E=(1-\operatorname{Ln} t)^{\wedge} 7 \\
& 1 / \sin 60=(1-\operatorname{Ln} t)^{\wedge} 7 \\
& t=102 \\
& v=d / t \\
& =(70+125+400+400) / 1.02 \\
& =975 \\
& t=K E=1 / 2 M v^{\wedge} 2 \\
& 102=1 / 2 M(.975)^{\wedge} 2 \\
& M=214.6 \\
& M=\operatorname{Ln} t \\
& t=1585=M o m e n t \\
& =F \times d=3 F \quad F=528
\end{aligned}
$$$$
\mathrm{PE}=\mathrm{Mc}^{\wedge} 2
$$$$
1 / \sin 60=M\left(c^{\wedge} 2\right)
$$$$
M=13.01
$$$$
\mathrm{t}=\mathrm{E}^{\wedge} \mathbf{2}+\mathrm{E}-\mathbf{2}
$$$$
=13^{\wedge} 2+13-2
$$$$
=180=\text { Pi Rads. }
$$

Figure3.

$$
\begin{aligned}
& \mathrm{t}=\mathrm{KE}=1 / 2 \mathrm{Mv}^{2} \\
& =(\mathrm{M} / 2) \mathrm{v}^{2} \\
& \mathrm{TE}=\mathrm{M}[1592] \\
& =\mathrm{M} / 2 \pi \\
& \mathrm{t} / \mathrm{TE}=\mathrm{v}^{2} /(1 / \pi)
\end{aligned}
$$


$=3.216^{2}(1 / \pi)$

$=32.92$

$=1 / 3.05$

$\mathrm{E}=(1-\ln \mathrm{t})^{7}$

$=(1-\ln 32.92)^{7}$

$=-6.357$

$\mathrm{t}=1 / \mathrm{E}=157.3=\pi / 2$

$\mathrm{t}=\mathrm{KE}=1 / 2 \mathrm{Mv}^{2}$

$=(\mathrm{M} / 2) \mathrm{v}^{2}$

$157.3=\mathrm{M} / 2(3.216)$

$=32.19$

$=1 / 31.06 \sim 31$ 12th Prime Number

$(31 / 12)^{2}=6.67=\mathrm{G}$

$\mathrm{t}=\mathrm{E}^{2}+\mathrm{E}-2$

$\mathrm{t}^{2}-\mathrm{t}-1$

Substitute:

$\mathrm{t}=\left(\mathrm{t}^{2}-\mathrm{t}-1\right)^{2}+\left(\mathrm{t}^{2}-\mathrm{t}-1\right)-2$

$t^{4}-2 t^{3}-2 t^{2}-3 t-1$

Take Derivative twice:

$4 t^{3} / 6-6 t^{2} / 2-4 t-3=0$

$12 \mathrm{t}^{2} / 9-12 \mathrm{t} / 4-4=0$

$\mathrm{t}^{2}-3 / 8 \mathrm{t}-3=0$

Quadratic:

$\mathrm{t}=\sqrt{ } 3 / 2 ;-1.372$

$\mathrm{t}=\sin 60^{\circ}$

$E=1 / \sin 60^{\circ}$

Now Integrate twice:

$\iint \mathrm{E} d \mathrm{t}=\iint\left(\left[1 / \sin 60^{\circ}\right]\right.$

$E^{3} / 3=1 / \sin 60^{\circ} \times \mathrm{t}^{2} / 2$

$(2 / \sqrt{ } 3)^{3}=\sqrt{ } 3 / 2 \cdot(3 / 2) t^{2}$

$\mathrm{t}=1.8856$

$\mathrm{t}^{2}-\mathrm{t}-1=\mathrm{E}$

$=6699 \sim G$

$\Rightarrow$ Clairnaut DE.

"Give me time and order, and I will give you design." [1]

$\mathrm{t}=\sqrt{3}$

Order $=\mathrm{G}=6.67$

$\mathrm{M}=\operatorname{Ln} \mathrm{t}=\operatorname{Ln} \sqrt{3}=549.3$

$\mathrm{s}=|\mathrm{E}||\mathrm{t}| \sin \theta$ 


$$
\begin{aligned}
& =\mathrm{Et}(1 / \mathrm{E}) \\
& =\mathrm{Et}^{2} \\
& =(1 / \sqrt{ } 3)(\sqrt{ } 3)^{2} \\
& =\sqrt{ } 3 \\
& =\mathrm{t} \\
& \mathrm{s}=\mathrm{t}
\end{aligned}
$$

\section{Clairnaut}

$\mathrm{d}^{2} \mathrm{E} / \mathrm{dt}^{2}-\mathrm{G}=0$

$\iint \mathrm{d}^{2} \mathrm{E} / \mathrm{dt}^{2}=\iint \mathrm{G} d \mathrm{t}$

$\mathrm{E}=\mathrm{G}^{3} / 3=(2 / 3)^{3 / 3}$

$=0.989$

$E=(1-\operatorname{Ln} t)^{7}$

$0.989=(1-\operatorname{Ln} t)^{7}$

$\mathrm{t}=2.53=$ Period $\mathrm{T}$

$\mathrm{E}=1 / \mathrm{t}=1 / 2.53=0.395 \sim 4$

Now,

$\mathrm{L}=\mathrm{Ln} \mathrm{t}+\mathrm{c}^{3}$

$\mathrm{E}=\left(1-\operatorname{Ln} \mathrm{t}+\mathrm{c}^{3}\right)^{7}$

$\mathrm{E}=\left(1-\operatorname{Ln} 1+3^{3}\right)$

$\mathrm{E}=28$

$\mathrm{t}=1 / 28=0.03571$

$=\{8$ choose 2$\}$

$=8 ! /(2 !)(6 !)$

$8 \times 7 / 2=28$

$\mathrm{M}=\mathrm{Ln} \mathrm{t}$

$=\operatorname{Ln}(.03571)$

$=0.333$

$\mathrm{M}=1 / \mathrm{c}$

\section{Poisson's Ratio:}

$v=0.2598 \sim 26$ Refer to Figure 2

$\varepsilon_{\mathrm{H}} / \varepsilon_{\mathrm{V}}$

$=(\Delta \mathrm{L} / \mathrm{L})_{\mathrm{H}} /(\Delta \mathrm{L} / \mathrm{L})_{\mathrm{V}}$

$=0.26$

$(1 / 2) /(1 / \sqrt{ } 3)$

$=0.866$

$=\sin 60^{\circ}$

\section{$0.26 / 0.866=2.999=c$}

$\mathrm{t}=\mathrm{E}^{2}+\mathrm{E}-2$

$\mathrm{E}=1 / \sin 60^{\circ}$ 


$$
\begin{aligned}
& \mathrm{t}=(1 / 0.866)^{2}-(1 / 0.866)-2 \\
& =1.333+1 / \sin 60^{\circ}-2 \\
& =-1.821 \\
& \mathrm{E}=1 / \mathrm{t}=-0.549 \\
& \mathrm{t}^{2}-1.5 \mathrm{t}-3=\mathrm{E} \\
& (\sqrt{ } 3)^{2}-1.5(\sqrt{ } 3)-3=\mathrm{E} \\
& \mathrm{E}=-0.2598 \sim 0.26=\text { Poisson's Ratio }
\end{aligned}
$$

Comprehension, far from being a Godlike talent which all design must follow, is an emergent effect of systems of uncomprehending competence; natural selection on the one hand; and mindless computation on the other. Pg 75 [1]

\section{Entropy:}

$\mathrm{d}^{2} \mathrm{E} / \mathrm{dt}^{2}-\mathrm{G}=0=\mathrm{S}=\Delta \mathrm{Q} / \Delta \mathrm{T}$

$\iint \mathrm{d}^{2} \mathrm{E} / \mathrm{dt}^{2}=\iint \mathrm{G}$

$\mathrm{E}=\mathrm{G}^{3} / 3+\mathbb{C}$

$\mathrm{E}=0.9891+\mathbb{C}$

$=\Delta \mathrm{Q} / \Delta \mathrm{T}$

$0.9891+\mathbb{C}=\Delta \mathrm{Q} /(273.15+20)$

$\Delta \mathrm{Q}=292.39+292.15(\mathbb{C})$

$\Delta \mathrm{Q}=\Delta \mathrm{E}=292.15+292.15 \mathbb{C}$

$\Delta \mathrm{E}=1 / \sqrt{3}-1 / \sin 60^{\circ}=292.15+292.15 \mathbb{C}$

$2886=292.15+292.15 \mathbb{C}$

$\mathbb{C}=-1$

$\Delta \mathrm{Q}=292.15+292.15(-1)$

$=\mathbf{0}=\Delta \mathbf{E}$

$=\operatorname{Ln} 1$

$=\mathrm{Ln} \mathrm{t}=\mathrm{M}$

$\mathrm{M}=\Delta \mathrm{E}=\operatorname{Ln}(\sqrt{3})$

$\mathrm{TE}=\mathrm{M}[0.1592]$

$\mathrm{TE}=\mathrm{E}(1 / 2 \pi)$

$=\operatorname{Ln} \sqrt{ } 3(1 / 2 \pi)$

$=0.1748 \sim 175$

$=57.19 \alpha 1 \mathrm{rad}$

$\mathrm{s}=\mathrm{t}=\mathrm{E}=1$

$\mathrm{s}=\mathrm{E}(1) \sin 60^{\circ}$

$1 / \sin 60^{\circ}=\mathrm{E}$

Vol. $=4 / 3 \pi \mathrm{R}^{3}$

Vol. $=2 \pi \mathrm{R}^{2}$

$=2 \pi(1)^{2}$

$=2 \pi$ 
$\mathrm{TE}=\mathrm{M}\{0.1592]$

$\mathrm{TE}=\mathrm{M} \times 1 /$ Vol'

$\mathrm{TE}=\rho=$ Density

$\mathrm{TE}=-1=\mathrm{M}(1 / 2 \pi)$

$\mathrm{M}=-2 \pi$

$\mathrm{M}=-\mathrm{Lx} \mathrm{t}$

$\mathrm{d}^{2} \mathrm{E} / \mathrm{dt}^{2}-\mathrm{G}=\mathrm{S}$

$\iint \mathrm{d}^{2} \mathrm{E} / \mathrm{dt} \mathrm{t}^{2}=\iint(\mathrm{S}+\mathrm{G}) \mathrm{dt}$

$\mathrm{E}=\mathrm{st} / 2+\mathrm{Gt}^{2} / 2+$

$E=(0+6.67) t^{2} / 2+\mathbb{C}$

$\mathrm{E}=\mathrm{t}^{2} / 2+\mathbb{C}$

$2=\mathrm{t}^{2} \cdot 1 / \mathrm{E}+\mathbb{C}$

$2=\mathrm{t}^{3}+\mathbb{C}$

$\mathrm{t}^{3}=2+\mathbb{C}$

$\mathbb{C}=-1$

$t^{3}=1$

$t= \pm 1$

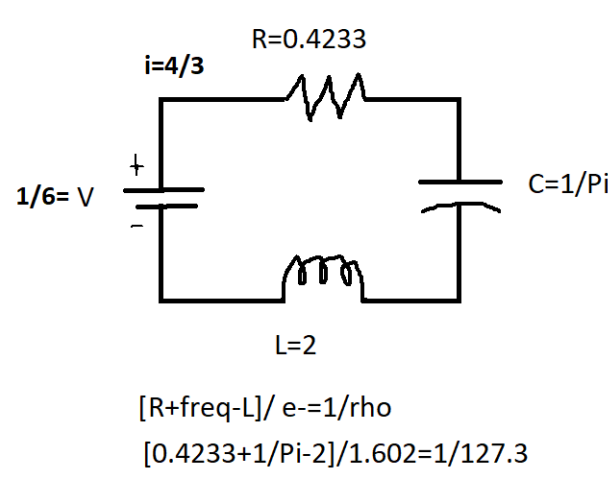

Figure 4

Mom=F x d

From above:

$\mathrm{F}=528$

Mom=528 x 3

$=1584$

$=$ Moment $=\mathrm{t}$

$E_{1} / E_{2}=1 / \sqrt{ } 3=2 \sin 30^{\circ} /\left[2 \sin 60^{\circ}\right]=1(1 / 2) /[2(\sqrt{ } 3 / 2)]$

$\mathrm{E}=2 \sin \theta=2 \sin 30=1$

$\mathrm{d} \theta / \mathrm{dt}=60^{\circ} / \mathrm{dt}=60^{\circ} / 1584=(\pi / 3) / 1584=59.5$

\section{Coriolis}

$\alpha=\omega^{2} \mathrm{R}$

$=(59.5)(2)$

International Journal of Scientific and Innovative Mathematical Research (IJSIMR) 
$=708 \sim 707=1 / \sqrt{ } 2$

$=\sin 45^{\circ}=\cos 45^{\circ}$

$\alpha=\omega^{2} \mathrm{R}$

$=(\mathrm{d} \theta / \mathrm{dt})^{2} \mathrm{R}$

$1 / \sqrt{2}=(\mathrm{d} \theta / \mathrm{dt})^{2}(2)$

$\mathrm{d} \theta / \mathrm{dt}=1 \mathrm{rad} / \mathrm{sec}$

$(1 / \sqrt{ } 2)^{2}+(1 / \sqrt{ } 2)^{2}=1$

$\sin ^{2} \theta+\cos ^{2} \theta=1$

$2 \sin ^{2}=1$

$2 \sin \theta=1 / \sin \theta=1$

$\mathrm{E}=2 \sin \theta=2 \sin 60^{\circ}$

$\mathrm{E}_{1}=\sqrt{3}$

$\mathrm{E}=2 \sin \theta=2 \sin 30^{\circ}$

$\mathrm{E}_{2}=1$

$E_{2} / E_{1}=1 / \sqrt{ } 3=$ Universal signal

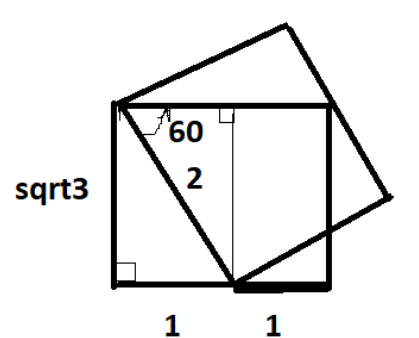

Rotate right 60 degrees

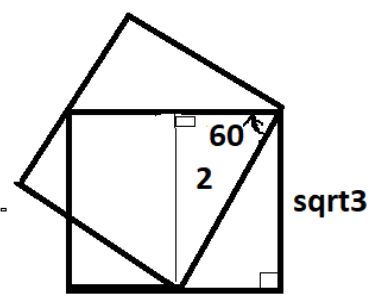

1

1

Rotate left 60 degrees

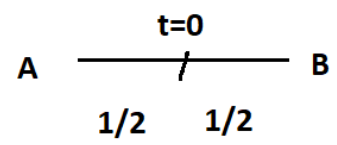

Figure 5

$\sin ^{\wedge} 260+\cos ^{\wedge} 260=1$

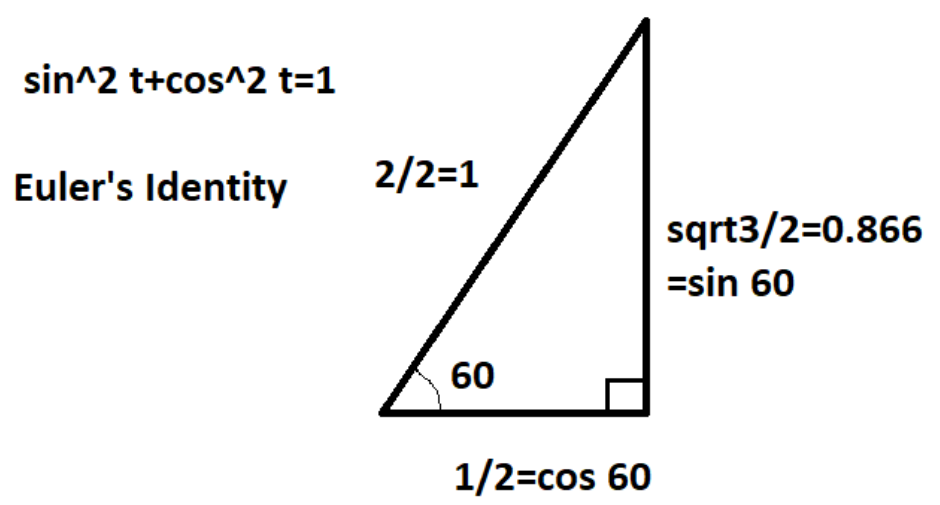

Figure 6 
The solution to the illusion of the Necker Cube representing the physical universe is summed up in Euler's Identity. $\sin ^{2} \theta+\cos ^{2} \theta=1$

There is an old problem in Math, and it goes like this: If we are at point A and go half way toward point B, we still have half way to go. Then if we go halfway again, we still half that much to go. If you can always take half of the remained, how do we ever get to point B?

The answer is that the motion is relative. As we go halfway from point $A$ to point $B$, point $B$ goes half wat to point $A$. They meet in the middle at $t=0$. In terms of the physical universe, this is when $E=t=1$ and $E=1 / \sqrt{ } 3$ and $t=\sqrt{ } 3$.

Water funnelling down a drain in counterclockwise in the norther hemisphere and clockwise in the southern hemisphere. Here why:

$\alpha=\omega^{2} \mathrm{R}$

$(0.595)^{2}(2)$

$=0.707 \sim 1 / \sqrt{ } 2=\sin 45^{\circ}=\cos 45^{\circ}$

2. EULER'S EQUATION

$\sin ^{2} 45^{\circ}+\cos ^{2} 45^{\circ}=1$

$\overline{\mathrm{F}}+\overline{\mathrm{P}}=1$

$\mathrm{M}(\mathrm{a}+\mathrm{v})=1$

$\mathrm{M}=(1) /(\mathrm{a}+\mathrm{v})$

$\mathrm{M}=1 /(1 / \sqrt{ } 2+1 / \sqrt{ } 2)$

$\mathrm{M}=1 /(\sqrt{ } 2)$

$\mathbf{M}=\mathbf{0 . 7 0 7}$

$\mathrm{TE}=\mathrm{M}[0.1592]$

$=(\sqrt{ } 2)(1 / 2 \pi)$

$=0.318$

$=1 / \pi$

=freq of the human mind.

$\mathrm{E}=1 / \mathrm{t}$

freq of the universe $=1 / \sqrt{ } 3$

freq $\times \mathrm{E}=\mathrm{h}=6.626$

$1 / \sqrt{3} \times \mathrm{E}=6.626$

$\mathrm{E}=1147$

Freq of the Brain $=1 / \pi$

freq $\times \mathrm{E}=6.6236$

$1 / \pi \times \mathrm{E}=6.626$

$\mathrm{E}=20.8$

$\mathrm{E}_{\mathrm{U}} / \mathrm{E}_{\mathrm{B}}=1.147 / 2.08=23.87$

Ln $23.87=3.173 \sim 1 / \pi$

Now,

freq $=1 / \pi=1 / \mathrm{t}$

But freq $=1 / \mathrm{T}=1 /(1 / \mathrm{t})=\mathrm{t}$

So, $\mathrm{t}=1 / \mathrm{t}$ 


\section{$\mathrm{t}^{2}=1$ \\ $\mathrm{t}= \pm 1$}

The Mind:

$\mathrm{L} x$ freq $=2(1 / \pi)=2 / \pi=6.366=\mathrm{t}_{0}$

$6.366 / 2=3183=1 / \pi=$ freq of the human mind begins at $t_{0}$ (ie Creation.)

So, I have shown in previous papers that we have no need for a Creator (or Designer); a Soul; nor a Heaven. But there is prophecy. Prophecy involves knowing the future or making it happen. We will assume that humans cannot make it happen, so there must be some sort of time travel. We know that the human mind is governed by the "Mind triangle. "See figure below.

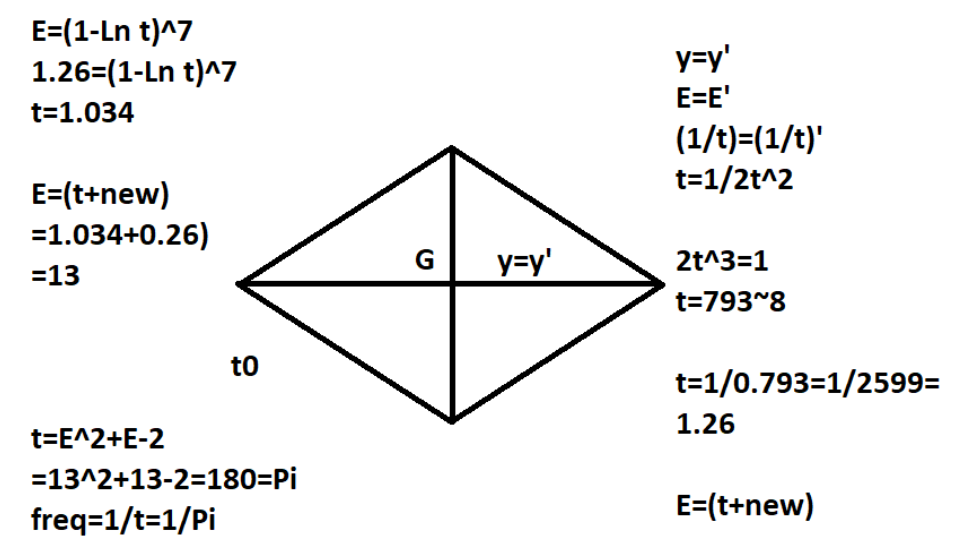

Figure 6

\section{Human DNA}

$2.15 \times 1.67$ Dalton $=\mathbf{3 6 . 5 5} \mathbf{g m}$

$\mathrm{M}=\mathrm{Ln} \mathrm{t}$

$36.55=\operatorname{Ln} \mathrm{t}$

$\mathrm{t}=12.96 \sim 13$

$\mathrm{t}=\mathrm{E}^{2}+\mathrm{E}-2$

$=13^{2}+13-2$

$=180=\pi$

$\mathrm{E}=(1-\mathrm{Lnt})^{7}$

$=(1-\operatorname{Ln} \pi)^{7}$

$=157.5 \sim \pi / 2=\mathrm{t} / \mathrm{L}$

$\mathrm{L}=\mathrm{Ln} \mathrm{t}+\mathrm{c}^{3}$

$\mathrm{L}=(2 / \pi) \mathrm{t}=\ln \mathrm{t}+27$

Derivative:

$2 / \pi=1 / \mathrm{t}+27$

$6.366-27=1 / \mathrm{t}$

$33.366=1 / \mathrm{t}$

$\mathbf{t}=\mathbf{3}$

Game of Life Physics

$\{8$ choose 2$\}=8 ! /\{2 ! \times 6 !\}=28 \Rightarrow 1$ Or 0 
$\{8$ choose 3$\}=56 \Rightarrow 1$

So,

$1 \times 28=28$

0x $28=0$

$1 \times 56=56$

$28+56=84$

\section{$56 / 84=6.66=G$}

\section{Order}

$\mathrm{y}=\mathrm{y}{ }^{\prime}=\mathrm{Gt}$

$=2 / 3(3)$

$=2 \Rightarrow \mathrm{SE}=\mathrm{SE}$ '

$\mathrm{t}=3 \mathrm{E}=5$

If $y=2 y^{\prime}=0$

If $y=3 \quad y^{\prime}=1$

$y=2+3=5 \quad y^{\prime}=1+0=1$

$\int \mathrm{y}^{\prime} \mathrm{dt}=\mathrm{t}+\mathbb{C}$

$\mathrm{y}=\mathrm{y}^{\prime}=5+\mathbb{C}=\mathrm{E}$

$\mathrm{Gt}=(2 / 3)(1 / 5)=2 / 15=0.1333=\mathrm{s}=\mathrm{t}$

$\mathrm{Gt}=(2 / 3)(3)=2$

$\mathrm{t}=2(0.1333)=\mathbf{2 . 6 6 6}=\mathrm{SF}$

$\mathrm{t}^{2}-\mathrm{t}-1=\mathrm{E}$

$\mathrm{E}=1 / \sin 60^{\circ}=115.47$

$\mathrm{t}^{2}-\mathrm{t}-1=115.47$

$\mathrm{t}^{2}-\mathrm{t}-2.1547=0$

$\mathrm{t}=15.181 / \mathrm{t}=\mathrm{E}=0.709 \sim \mathbf{1} / \sqrt{ } \mathbf{2}=\alpha$

This leads to Euler's Identity, and thus the Necker's Cube.

\section{CONCLUSION}

The universe is chiral. It is both left and right-handed. Of course, your left hand is controlled by the right brain; and vice verse. So why is it left and right-handed? It is neither. It is both at once. The solution to the optical illusion of the Necker Cube is Euler's Identity.

\section{REFERENCES}

[1] Dennett., DC., From Bacteria to Bach and ack The Evolution of minds., Norton NY., 2017.

Citation: PAUL T E CUSACK, Necker's Cube and the Universe, International Journal of Scientific and Innovative Mathematical Research (IJSIMR), vol. 8, no. 8, pp. 11-21, 2020. Available : DOI: https://doi.org/10.20431/2347-3142.0808002

Copyright: (C) 2020 Authors. This is an open-access article distributed under the terms of the Creative Commons Attribution License, which permits unrestricted use, distribution, and reproduction in any medium, provided the original author and source are credited. 